\title{
IL FORUM DI MM
}

Chiarissimo Direttore,

la volontà espressa nell'ultimo numero di Microbiologia Medica di aprire a tutti i soci dell'AMCLI la possibilità di partecipare le proprie opinioni, i propri pareri, le proprie esperienze in questo campo del tutto peculiare della Medicina di Laboratorio, con la ferma intenzione di trasformare la rubrica del Supplemento "L'Opinione" (supplemento allegato alla Rivista medesima) in un "Forum di Microbiologia Clinica", mi trova d'accordo e in perfetta sintonia.

Da tempo si lamentava infatti la mancanza di una "rubrica libera ed aperta", in altre parole di "Lettere al Direttore" (per usare un aforisma datato ma pur sempre utile e prezioso), che dovrebbe e/o potrebbe supportare sia la nostra Rivista che il periodico notiziario dell'Associazione, non sempre finalizzato a ciò, nel fornire non soltanto suggerimenti di carattere opinionistico su vari aspetti della nostra "difficile" disciplina, ma altresì notizie ed informazioni di interesse scientifico che possano essere esplicative, anche, del ruolo fondamentale che il Microbiologo Clinico svolge nella diagnosi delle malattie infettive e da infezione (siano esse batteriche, fungine, virali o parassitari).

Sperando di fare cosa gradita e di essere di stimolo per gli altri colleghi, che non sono pochi, mi preme riportare il "nostro" primo caso di enterite da Campylobacter upsaliensis, ed uno dei primi segnalati in Italia, anche per "documentare" l'importanza delle "nostre" figure professionali. In altre parole tale "Caso clinico", che viene riportato in questo medesimo numero di Microbiologia Medica, vuole rappresentare un esempio di un percorso diagnostico attento, razionale e corretto per una diagnostica importante, quale quella delle infezioni intestinali, che sovente viene "banalizzato" e che invece è bagaglio fondamentale della nostra disciplina.

Ecco pertanto che anche il neonato "Forum" può essere tavolo di dibattito per garantire un confronto aperto in merito agli annosi problemi che riguardano non soltanto la nostra professione ma più ancora le nostre figure professionali nel campo "pieno di insidie, di incomprensioni e di sopraffazioni" della medicina di laboratorio.

Ringrazio dell'attenzione,

Daniele Crotti, Libero Professionista in Parassitologia e Microbiologia Medica Strada Comunale per Pilonico Paterno, 4 06080 Pianello, Perugia 